\title{
Maldivas y el delicado equilibrio entre India y China
}

\section{Matías Iglesias ${ }^{1}$ \\ Universidad de Palermo}

\section{Ensayo}

Material original autorizado para su primera publicación en el Journal de Ciencias Sociales, Revista Académica de la Facultad de Ciencias Sociales de la Universidad de Palermo.

\section{Recepción: 17-09-2018}

\section{Aceptación: 04-02-2019}

Resumen: Maldivas es la nación más pequeña de Asia Meridional en términos de población, superficie y PBI. A pesar de ello, este pequeño archipiélago tiene una gran importancia estratégica, en virtud de su ubicación en medio de las rutas marítimas internacionales del océano Índico, y en su cercanía a las costas de la India. La propuesta de este artículo es exponer los cambios que se produjeron en Maldivas durante la presidencia de Abdulla Yameen en cuanto a la política interna, como así también a las relaciones exteriores, y observar en qué momento estas dos tendencias se cruzan para formar parte de una misma trama. A su vez, analizar finalmente las consecuencias que esto tiene para el país y para la región del Océano Índico. Numerosos observadores destacan que desde la llegada al poder de Abdulla Yameen la política interna está signada por el deterioro de la joven democracia de Maldivas. Al mismo tiempo, el presidente Yameen ha estrechado fuertes lazos políticos y económicos con la República Popular China. La convergencia de estas dos tendencias se produjo a principios de 2018, cuando la crisis política en Maldivas y la declaración del estado de emergencia dio lugar a especulaciones sobre una posible intervención militar por parte de India. Rápidamente China se posicionó en contra de tal posibilidad en nombre de la soberanía y la no injerencia. La situación de Maldivas representa un desafío grave para la India, puesto que abre la posibilidad para una futura base naval china en el archipiélago, a pocas millas de sus propias costas. La regresión democrática, además, es en sí misma un desafío para el estatus y la credibilidad de India frente a los otros Estados de su vecindario.

Palabras clave: Maldivas; India; China; océano Índico.

\footnotetext{
${ }^{1}$ Licenciado en Relaciones Internacionales (UP); Magíster en Geopolítica (Universidad Carolina, Praga). Correo electrónico: matiasiglesias09@gmail.com
} 


\section{Maldives and the delicate balance between India and China}

Abstract: Maldives is the smallest nation in South Asia in terms of population, area and GDP. Despite this, this small archipelago has great strategic importance, due to its location in the middle of international sea lines of communication in the Indian Ocean, and its proximity to the Indian mainland. The proposal of this article is to expose the changes that took place in the Maldives during the presidency of Abdulla Yameen in terms of internal politics and external relations, observing at what moment these two tendencies cross to be part of the same plot, and finally to analyze the consequences this has for the country and for the Indian Ocean Region. Numerous observers point out that since Abdullah Yameen came to power, domestic politics has been characterized by the deterioration of the young Maldivian democracy. At the same time, president Yameen has established strong political and economic ties with the People's Republic of China. The convergence of these two trends occurred at the beginning of 2018, when the political crisis in the Maldives and the declaration of a state of emergency gave rise to speculation about a possible military intervention by India. China quickly positioned itself against such a possibility in the name of sovereignty and non-interference. The situation in the Maldives represents a serious challenge for India, since it opens the possibility for a future Chinese naval base in the archipelago, a few miles from Indian mainland. The democratic regression, moreover, is by itself a challenge to the status and credibility of India vis-à-vis the other States in its own backyard.

Key words: Maldives; India; China; Indian Ocean.

\section{Introducción}

Maldivas, un archipiélago de alrededor de 1200 islas, de las cuales 200 se encuentran habitadas, es la nación más pequeña de Asia Meridional en términos de población, superficie y PBI. Es el país más bajo del mundo con un promedio de 1,5 metros sobre el nivel del mar, y el que presenta la altitud máxima menos elevada del mundo: 2,3 metros, lo cual deja al país particularmente vulnerable a la subida del nivel del mar.

Naturalmente, la pesca ha sido la principal actividad de la economía y aún hoy es la principal ocupación de la población, aunque en los últimos años el turismo ha crecido y constituye el mayor ingreso de moneda extranjera que percibe el país. Si acaso el lector ha oído hablar de Maldivas, probablemente haya sido en relación con su vulnerabilidad al cambio climático o bien con su perfil turístico.

No obstante, esta pequeña nación insular ha tenido tanto históricamente como en la actualidad una gran importancia estratégica en virtud de su ubicación en medio de las rutas 
marítimas internacionales del océano Índico, y en su cercanía a las costas de la India (Rajagopalan, 2018).

En vista de esta importancia estratégica, la propuesta de este artículo es exponer los cambios que se produjeron en Maldivas durante la presidencia de Abdulla Yameen en cuanto a la política interna, como así también a las relaciones exteriores, y observar en qué momento estas dos tendencias convergen para formar parte de una misma trama. A su vez, analizar finalmente las consecuencias para el país y para la región del Océano Índico.

Numerosos observadores destacan que, desde la llegada al poder de Abdulla Yameen, la política interna está signada por el deterioro de la joven democracia de Maldivas (Blomqvist, 2018; Human Rights Watch, 2018; Radhakrishnan, 2016; Ramachandran, 2018b). Al mismo tiempo, el presidente Yameen ha complementado su maniobra interna de consolidación de poder por medio de un acercamiento estratégico con la República Popular China y un distanciamiento de India (Dhar, 2018; Kumar, 2015; Ramachandran 2018; Tiezzi, 2018).

La convergencia de estas dos tendencias se produjo a principios de 2018, cuando la crisis política en Maldivas y la declaración del estado de emergencia dio lugar a especulaciones sobre una posible intervención militar por parte de India. Rápidamente China se posicionó en contra de tal posibilidad en nombre de la soberanía y la no injerencia (Rajagopalan, 2018; Tiezzi, 2018).

En la siguiente sección presentaré el marco teórico del trabajo. No se puede hablar de Maldivas sin ubicarse en el contexto de la Región del Océano Índico, la Iniciativa del Cinturón y la Ruta de la Seda y la teoría geopolítica del "collar de perlas". A continuación, abordaré la presidencia de Yameen desde una perspectiva histórica, destacando de manera especial los cambios e inflexiones más relevantes de la política interna y las relaciones exteriores. Luego haré referencia a la crisis de 2018, la posibilidad de una intervención por parte de India y la respuesta de China ante tal hipótesis, poniendo así de manifiesto el punto de encuentro entre la política interna y las relaciones exteriores de Maldivas durante la presidencia de Yameen. Finalmente, extraeré las conclusiones con respecto a Maldivas y la región del Océano Índico, en particular, para la India.

\section{La Región del Océano Índico: ¿Ruta Marítima de la Seda o collar de perlas?}

En su libro Monsoon: the Indian Ocean and the Future of American Power, el autor Robert D. Kaplan (2010) comienza diciendo que el siglo XX estuvo definido por el mapa de Europa: la política de las grandes potencias, desde el colapso de los imperios del Viejo Mundo hasta la lucha bipolar entre Estados Unidos y la Unión Soviética tuvo mayoritariamente que 
ver con el mapa europeo. El autor considera que, en el siglo XXI, el mapa del Océano Índico puede ser tan fundamental para las relaciones internacionales como lo fue el mapa de Europa en el siglo XX.

La significancia de la Región del Océano Índico es en cualquier caso insoslayable. Aun hoy en día, el $90 \%$ del comercio global y dos tercios de los suministros de petróleo en el mundo viajan por mar. El Océano Índico, cuyo volumen de navegación comercial se ha cuadruplicado desde 1970, representa la mitad del tráfico mundial de contenedores y la mitad del comercio mundial de petróleo. El Índico alberga no solo las principales líneas de transporte marítimo de petróleo, sino también los principales cuellos de botella marítimos del comercio mundial: los estrechos de Bab el-Mandeb, Hormuz y Malaca (Kaplan, 2010).

La seguridad en la Región del Océano Índico es importante para todas las naciones que se encuentran en sus costas, como así también para todas aquellas naciones que dependen del sistema de comercio marítimo global y usan el Océano Índico como pasaje (Singh, s.f.).

Esto es particularmente cierto para India y China. En efecto, a medida que millones de indios y chinos se sumen a la clase media global, su demanda de energía aumentará aún más. Para 2020, más del 85\% del petróleo con dirección a China pasará a través del Océano Índico y del estrecho de Malaca. Esta dependencia constituye una vulnerabilidad estratégica a la cual se hace referencia usualmente como el "dilema de Malaca” (Kaplan, 2010).

En 2013, el presidente chino Xi Jinping anunció la Iniciativa de la Franja y Ruta de la Seda (One Belt One Road - OBOR), que consiste en una parte continental, el Corredor Económico de la Ruta de la Seda, y su contraparte marítima, la Ruta Marítima de la Seda. Esta última es una estrategia de desarrollo por parte de China para, supuestamente, impulsar la conectividad de la infraestructura a través del Sureste Asiático, Oceanía, el Océano Índico y África Oriental (Green, 2018).

Muchas naciones han dado la bienvenida a la oportunidad de postularse para el financiamiento de proyectos por parte de China. Al mismo tiempo, surgen preguntas sobre la viabilidad económica y, en consecuencia, sobre las intenciones geopolíticas detrás de las propuestas de China. Hasta ahora, las iniciativas de la Ruta Marítima de la Seda se han concentrado principalmente en proyectos de desarrollo de puertos, lo que lleva a muchos observadores a preguntarse si estas inversiones son de naturaleza económica o militar, o ambas, y si, además, estas inversiones en gran escala no están estructuradas para que los países receptores, mediante una así llamada "trampa de deuda", terminen vulnerables a la presión ejercida por China sobre sus políticas domésticas y exteriores (Green, 2018). 
En cuanto a la naturaleza dual de los proyectos de desarrollo de puertos, vale hacer referencia a la teoría geopolítica americana conocida como "collar de perlas", un término usado por primera vez en 2004 en un reporte del Departamento de Defensa de los Estados Unidos, para referirse al acceso de China a bases en el Océano Índico. El reporte sugería que, en caso de un conflicto armado entre China y una o varias potencias, la presencia de la flota china en el Océano Índico podría disuadir la disrupción potencial de las líneas de abastecimiento de energía chinos por parte de sus enemigos, incluyendo la marina americana. Por su parte, India también ve con preocupación que el así llamado "collar de perlas" en el Índico le confiera a Beijing la posibilidad de escalar horizontalmente en el caso de un eventual enfrentamiento directo, por ejemplo, en los Himalayas (Green, 2018).

Con este contexto puesto sobre la mesa, comenzamos a hablar de Maldivas.

\section{Historia de Maldivas antes de 2013}

La historia de Maldivas es muy extensa y una exposición detallada excede el propósito de este artículo. Mencionaremos que el budismo llegó a las islas en el siglo III a.C., pero el islam, que fue introducido en 1153, es la religión predominante y oficial en la actualidad. En ese entonces se creó el Sultanato de Maldivas, que se convirtió en colonia portuguesa en 1558, holandesa en 1654 y británica en 1887. Finalmente, llegó la independencia en 1965, y tres años después el sultanato fue abolido de facto.

Desde entonces, Maldivas es una república presidencialista: en el poder ejecutivo se encuentra el presidente, quien es jefe de estado y de gobierno. El presidente (junto con el vicepresidente) es elegido directamente por el pueblo por un período de cinco años y se permite una reelección. El poder legislativo es ostentado por la Majlis, una legislatura unicameral compuesta de 85 miembros elegidos directamente para servir durante 5 años. En cuanto al poder judicial, el sistema legal está basado en la sharía. La Suprema Corte está compuesta por cinco miembros, elegidos por el presidente en consulta con la comisión de servicio judicial.

Desde 1978 a 2008 el país fue gobernado por Maumoon Abdul Gayoom, elegido presidente en 1978 y reelecto en 1983, 1988, 1993, 1998 y 2003, siendo al final de su última presidencia el mandatario con más tiempo en el poder en Asia. Durante ese período se fortaleció la relación contemporánea con India, quien jugó un rol crítico en la construcción de la economía moderna de Maldivas y aseguró la estabilidad política, permitiéndole a Gayoom quedarse en el poder durante tres décadas. En 1988, incluso, India despachó tropas al archipiélago para contrarrestar un intento de golpe contra el presidente: la Operación Cactus. Gracias a ese esfuerzo exitoso y apreciado, la India retuvo su influencia en el país por otras tres décadas (Panda, 2018; Ramachandran, 2018a). 
En 2008 tuvieron lugar las primeras elecciones verdaderamente democráticas con las cuales llegó al poder el hasta entonces opositor Mohamed Nasheed (HRW, 2018). Habíamos mencionado que Maldivas es particularmente vulnerable a las consecuencias del cambio climático, en particular, la subida del nivel del mar. Un episodio recordado de la presidencia de Nasheed tuvo lugar en octubre de 2009: como parte de una campaña de concientización sobre las amenazas del cambio climático y sus efectos sobre Maldivas, Nasheed encabezó la primera reunión de gabinete bajo el agua en el mundo, con los participantes acudiendo a la cita en equipo de buceo (Reddy, 2009).

Desde el primero de mayo de 2011, una serie de protestas pacíficas, motivadas principalmente por el descontento con la economía, fueron escalando hacia una crisis política terminal que dio como resultado la renuncia de Mohamed Nasheed el 7 de febrero de 2012. El vicepresidente Waheed Hassan Manik asumió el cargo y llamó a elecciones para 2013.

En la primera vuelta, el 7 de septiembre de 2013, el expresidente Nasheed obtuvo (irónicamente) la mayor cantidad de votos (aunque sin mayoría absoluta), pero la Suprema Corte anuló la elección. En la segunda vuelta se repitieron los resultados. Se celebró entonces una tercera vuelta de desempate entre Nasheed y Abdulla Yameen, medio hermano y delfín del expresidente Gayoom, quien tenía la segunda mayor cantidad de votos. En ese desempate, finalmente, Yameen obtuvo un pequeño margen y se alzó con la victoria. Asumió la presidencia el 17 de noviembre de 2013 (HRW, 2018).

\section{Presidencia de Yameen}

La presidencia de Abdulla Yameen estuvo caracterizada por dos tendencias paralelas en la política interna y externa. En la política interna, muchos observadores reportan que la democracia de Maldivas se ha visto sensiblemente deteriorada a causa de la búsqueda de acumulación y concentración del poder por parte del presidente (Radhakrishnan, 2016; Ramachandran, 2018b). En la política externa, se ha producido un distanciamiento de India y se han estrechado lazos notorios con la República Popular China (Dhar, 2018). Estas dos tendencias convergieron en la crisis política de principios de 2018, cuando el presidente Yameen declaró el estado de emergencia en Maldivas. A continuación, se exponen ambos aspectos por separado.

\subsection{Política interna: deterioro de la democracia}

Uno de los hitos más importantes en el declive democrático del país ocurrió en febrero de 2015, cuando el expresidente Mohamed Nasheed fue arrestado y condenado a 13 años de prisión por cargos de terrorismo. El juicio demoró menos de veinte días y el equipo legal 
de Mohamed Nasheed no pudo convocar testigos propios. Dos de los tres jueces del caso fueron testigos para la querella (Radhakrishnan, 2016; Ramachandran, 2018b).

El Grupo de Trabajo sobre Detenciones Arbitrarias de Naciones Unidas se expresó contra el proceso y urgió a la liberación inmediata de Nasheed. Bajo presión internacional, en enero de 2016 se le otorgó a Nasheed un permiso para viajar al Reino Unido por una operación de espalda. En mayo de 2016, el gobierno británico le otorgó estatus de refugiado y desde entonces Nasheed ha permanecido en el exilio. En la actualidad se encuentra en Sri Lanka (Blomqvist, 2018; HRW, 2018).

Sus seguidores políticos y los miembros de su partido, mientras tanto, han sido objeto de persecución en Maldivas. También se encuentran en prisión un ex ministro de defensa, el Cnel. Nazim, líder del partido Adhaalat de la oposición, así como el Sheik Abdullah y el exvicepresidente Ahmed Adheeb, todos condenados por períodos mayores a una década (Radhakrishnan, 2016; Ramachandran, 2018b).

En la segunda mitad de 2017, se produjo una ruptura entre Yameen y su padrino político, el expresidente Gayoom, quien, como vimos, había gobernado el país durante 30 años. En consecuencia, doce parlamentarios que respondían a Gayoom se cambiaron de bloque en el parlamento, pasando del oficialismo a la oposición. Fueron despojados de sus bancas y encarcelados (Blomqvist, 2018; Ramachandran, 2018b). Como veremos más adelante, la Corte Suprema exigió su liberación, lo cual fue el puntapié de la crisis de 2018.

En general, la libertad de expresión y de prensa se han visto bajo amenaza. Los periodistas en Maldivas han enfrentado abusos, amenazas crecientes y en algunos casos ataques violentos, creando así un ambiente de miedo y autocensura (HRW, 2018).

El 11 de agosto de 2016 el presidente Yameen promulgó la Ley de Anti-Difamación y Libertad de Expresión, la cual criminaliza el discurso "difamatorio" así como también los comentarios contra "cualquier principio del islam", comentarios que "amenacen la seguridad nacional" o que "contradigan normas sociales generales" (HRW, 2018; Radhakrishnan, 2016).

La ley entró en vigor durante el escándalo de corrupción de una corporación estatal que involucraba el desfalco de aproximadamente 84 millones de dólares por medio de la venta y alquiler de islas. Los medios publicaron acusaciones de que oficiales en el gobierno y en la oposición se beneficiaron. El gobierno de Yameen, en vez de investigar los alegatos, arrestó al gerente del Banco de Maldivas que colaboró en develar la trama de corrupción, por exponer datos confidenciales de los clientes (HRW, 2018).

La Ley Anti-Difamación impone multas fuertes para medios, periodistas o individuos que sean encontrados culpables. Solo se puede apelar después de pagar la multa. Los medios que no pueden pagar pueden ver sus licencias suspendidas o revocadas. Los 
periodistas que no puedan pagar pueden enfrentar entre tres y seis meses de prisión. La carga de la prueba se invierte y los periodistas pueden tener que revelar sus fuentes, por cierto, en contradicción con la constitución (HRW, 2018).

En octubre de 2015, el gobierno promulgó la Ley Antiterrorismo a pesar de la crítica generalizada de la oposición y los observadores internacionales. Esta ley (así como otras anteriores que había en el país, por cierto) contiene definiciones de terrorismo muy vagas y tan amplias que cubren delitos comunes que no deberían razonablemente ser considerados terrorismo, o incluso actos que no son crímenes en absoluto, dejando lugar a aplicaciones de la ley arbitrarias y discriminatorias por parte de las autoridades (HRW, 2018).

Por otro lado, la Ley de Libertad de Asamblea Pacífica de 2013 requiere a los organizadores de asambleas que informen a la policía de cualquier reunión que se esté planeando, y la policía tiene discrecionalidad para otorgar el permiso. Además, hay restricciones con respecto a áreas designadas y acceso limitado a periodistas. En 2016, la ley se reformó para imponer aún más restricciones que, para todo propósito práctico, vacía de contenido el derecho a la libertad de asamblea (HRW, 2018). Otros avances contra la democracia se han reflejado en arrestos masivos y arbitrarios durante protestas, y una ley para restringir el acceso de partidos políticos a la contienda electoral, limitando así el pluralismo en el país (HRW, 2018).

La erosión de la democracia no ha pasado inadvertida en el exterior. La Commonwealth se reunió varias veces en 2016 para discutir sobre la situación de Maldivas y elaboró duras críticas, advirtiendo sobre la posibilidad de una suspensión. El gobierno de Maldivas se limitó a responder que estaba siendo tratado injustamente por el bloque. Finalmente, en octubre de 2016, Maldivas abandonó la Commonwealth (Mohamed, 2016; Olazábal, 2016; Safi, 2016).

\subsection{Política externa: acercamiento a China}

Hasta 2011, China ni siquiera tenía embajada en Malé, pero la situación ha cambiado muchísimo desde entonces (Ramachandran, 2018a).

Una compañía de India, GMR Male International Airport Limited (GMIAL) estaba desarrollando un aeropuerto cerca de la capital. En 2012, el partido de Yameen (quien todavía no era presidente) calificó al acuerdo y al contrato, que había sido aprobado por el presidente anterior Mohamed Nasheed, como una venta de la soberanía de la nación (Kumar, 2015). En noviembre de ese año, con Nasheed ya fuera de la presidencia, el contrato fue cancelado abruptamente. La concesión se otorgó luego a una compañía estatal china. GMIAL llevó el caso a un tribunal arbitral internacional, el cual ordenó una compensación millonaria. Esta fue 
pagada prontamente, pero en India se sospecha que fue China quien aportó el dinero (Pararajasingham, 2017; Ramachandran, 2018a, Ramachandran, 2018b).

Con Yameen en la presidencia, la tendencia favorable a China se acentuó considerablemente. En septiembre de 2014, el presidente chino se convirtió en el primer líder extranjero en visitar Malé luego de la inauguración del nuevo gobierno, y el primer jefe de Estado chino en visitar las Maldivas en la historia. Durante su visita, se acordó que las Maldivas se conviertan en un socio oficial de la Ruta Marítima de la Seda (Kumar, 2015). A cambio, China comenzó a invertir en proyectos de infraestructura en las islas, incluyendo el Puente de la Amistad China-Maldivas entre Malé y Hulhumalé (donde se encuentra el aeropuerto), y un proyecto inmobiliario de más de 1000 apartamentos en Hulhumalé. Eventualmente, además, China reemplazaría a Europa como el origen del número más grande de turistas que visitan las islas (Pant, 2018; Ramachandran, 2018a).

En 2015, la Majlis aprobó una reforma histórica autorizando la propiedad extranjera de la tierra en cualquiera de las 1200 islas que componen el archipiélago, siempre y cuando la inversión sea superior a un billón de dólares y el 70\% del proyecto se encuentre ubicado en tierras ganadas al mar. En cuanto al procedimiento, la reforma se aprobó sin debate, sin consulta y sin tiempo para que los legisladores estudien el borrador (Kumar, 2015).

Aunque la ley es de aplicación general, se entiende que está pensada a la medida de China, la principal beneficiada, pues es el único actor con la capacidad financiera para hacer inversiones de ese calibre. La ley puede facilitarle a China la vía para adquirir propiedad en las islas y construir un puesto de escucha o incluso una base naval (Ramachandran, 2018a). Los legisladores críticos de la ley manifestaron su temor a que se perturbe el equilibrio de poder entre India y China en la región. Eva Abdullah, una legisladora de la oposición, llegó a afirmar que el país se transformaría en una colonia china (citada en Kumar, 2015). Sugestivamente, en agosto de 2017, el gobierno de Maldivas permitió atracar en Malé a 3 buques de guerra chinos (Pararajasingham, 2017, Ramachandran, 2018a).

En diciembre de ese mismo año, durante una visita de cuatro días de Abdulla Yameen a Beijing, se firmó un tratado de libre comercio entre Maldivas y China, el primero en la historia de la nación insular, y el segundo de un país de Asia del Sur con China, luego de Pakistán. Además del tratado, Maldivas firmó un memorándum de entendimiento que la coloca oficialmente en la Ruta Marítima de la Seda. También se firmaron otros pactos que profundizan la cooperación bilateral en un conjunto de áreas, incluyendo la salud, el turismo, la tecnología y el cambio climático (Pant, 2018; Ramachandran, 2018a).

Bajo el tratado de libre comercio, China y Maldivas reducirán las tarifas de más del $95 \%$ de los bienes a cero. También hay un compromiso para la apertura del mercado de servicios, tales como las finanzas, la salud y el turismo, a inversores chinos, lo cual les 
permitirá operar hoteles, restaurantes, puertos deportivos, agencias de viajes y servicios de transporte (Ramachandran, 2018a).

Por otro lado, se espera que el tratado de libre comercio impulse la industria pesquera del país. En 2011, Maldivas dejó de ser calificado como País Menos Desarrollado (PMD) y se recategorizó como País de Ingresos Medios. En consecuencia, dejó de ser elegible para las reducciones de tarifas de su socio comercial más grande: la Unión Europea. Desde entonces, los exportadores de pescado de Maldivas han tenido que pagar una tarifa del $25 \%$ para entrar al mercado de la UE. El tratado de libre comercio con China les da una nueva oportunidad, abriéndoles el mercado de consumo más grande del mundo a tarifa cero (Ramachandran, 2018a).

Como en el caso de la reforma anterior, otra vez los miembros del parlamento, a pesar de los pedidos, no tuvieron acceso al documento del tratado para revisarlo antes de su aprobación. El gobierno permitió menos de una hora para el proceso parlamentario completo antes de aprobar el documento de más de mil páginas que contiene la letra del tratado (Panda, 2018; Pant, 2018; Ramachandran, 2018a).

Los críticos consideran que se trata de una trampa de deuda con China (Dhar, 2018). De acuerdo con Mohamed Nasheed, por ejemplo, el 70\% de la deuda externa de Maldivas ya está contraído con China (Pararajasingham, 2017). Los analistas de seguridad en India advierten a su vez que la nación insular puede quedar vulnerable a las demandas y la presión de Beijing, y señalan los casos precedentes de Sri Lanka y Pakistán (Ramachandran, 2018a).

\section{Crisis de 2018: la convergencia de la política interna y la exterior}

Durante la crisis de 2018 se produjo la convergencia de estas dos líneas aparentemente paralelas y se pudo ver cómo formaban parte en realidad de un todo coherente.

El 1 de febrero, la Corte Suprema de Maldivas ordenó la liberación de todos los presos políticos, entre ellos el expresidente Mohamed Nasheed (en el exilio en Sri Lanka) y los doce parlamentarios que habían sido despojados de sus bancas en 2017 cuando abandonaron el oficialismo para pasarse a la oposición. La Corte Suprema argumentó que sus juicios habían sido sesgados políticamente. El veredicto allanaba el camino para que Nasheed regrese de su exilio en Colombo y se presente a las elecciones presidenciales de septiembre de 2018 , pero también volvía plausible un voto de no confianza contra el presidente en la Majlis, dado que el regreso de los parlamentarios expulsados transformaba al oficialismo en minoría (Pant, 2018; Ramachandran, 2018b). 
El gobierno se limitó a ignorar la orden de la Corte Suprema y mandó a arrestar a dos de los jueces y a Maumoon Gayoom, por las dudas (Panda, 2018; Pant, 2018; Tiezzi, 2018). Acto seguido, los jueces que no fueron arrestados rescindieron el veredicto original (Blomqvist, 2018; Ramachandran, 2018b) y, eventualmente, los dos que habían sido arrestados fueron sentenciados a dos años en prisión.

La población reaccionó con protestas generalizadas en la capital Malé, y se produjeron enfrentamientos entre la policía y los manifestantes. El presidente decretó un estado de emergencia por 15 días y ordenó al ejército que resista cualquier orden de la justicia (Blomqvist, 2018; Panda, 2018; Pant, 2018). A fin de febrero, el presidente Yameen extendió el estado de emergencia durante otros treinta días (Kabir, 2018; Rajagopalan, 2018) y lo levantó finalmente el 22 de marzo.

Durante la crisis, voces significativas de la oposición, incluyendo a Mohamed Nasheed, se expresaron públicamente en favor de que India tome cartas en el asunto, incluso mediante una intervención armada (Dhar, 2018; Blomqvist, 2018; Kabir, 2018; Panda, 2018; Ramachandran, 2018b; Tiezzi, 2018).

Cuando la especulación por el curso de acción que tomaría India adquirió notoriedad en la prensa y los analistas, China no demoró en pronunciarse al respecto, y llamó a la comunidad internacional a respetar la soberanía de Maldivas y "no tomar acciones que puedan complicar aún más la situación" (Rajagopalan, 2018).

Así, el miércoles 7 de febrero de 2018, Geng Shuang, vocero del ministerio de exteriores chino, dijo en una conferencia de prensa:

Lo que está sucediendo dentro de las Maldivas es un asunto interno del país [...]. La comunidad internacional debería jugar un rol constructivo sobre la base de respetar la soberanía de Maldivas, en vez de complicar aún más la situación [...]. Creemos que el gobierno de Maldivas, los partidos políticos y la gente tienen la sabiduría y la capacidad para lidiar con la situación corriente por su cuenta. (Tiezzi, 2018)

A pesar de todo, no faltaron en India quienes se pronunciaron en forma favorable a la intervención (Maldives Independent, 2018; Ramachandran, 2018b), y recordaron la intervención de 1988 (Dhar, 2018). Sin embargo, la situación actual es fundamentalmente distinta de aquella. En ese entonces, la India pudo incluso cosechar elogios por una aplicación unilateral de poder duro para prevenir un golpe, pero el presidente Gayoom había requerido la intervención y los conspiradores eran solo un pequeño grupo de mercenarios. Esta vez, Yameen es expresamente apoyado por China, quien puede usar su poder de veto para bloquear cualquier resolución de mano dura por parte del Consejo de Seguridad de Naciones Unidas (Dhar, 2018; Kabir, 2018; Panda, 2018; Ramachandran, 2018b). 
India se encuentra en 2018 en un ciclo de elecciones estaduales que representan un anticipo de las nacionales de 2019, y aunque la posición oficialista parezca sólida, es de esperarse que Modi se incline por la aversión al riesgo en el frente de la política exterior (Kabir, 2018). Por todos estos motivos, la intervención militar india durante la crisis de principios de 2018 fue descartada (Ramachandran, 2018b).

Lo que quedó claro es que el acercamiento a China y las numerosas concesiones y gestos que se otorgaron a la gran potencia en ascenso le rindieron fruto al presidente Yameen. Cuando parecía que la crisis política se desbordaba y surgían rumores de una posible intervención extranjera, China apareció rápidamente como garante de la estabilidad de su gobierno. La política exterior de China se rige por el cumplimiento estricto de ciertos principios que rigen sin contemplar excepciones, notablemente la preservación de la soberanía, la integridad territorial y la no injerencia en asuntos internos (Oliva, 2008; Tiezzi, 2018). Visto en retrospectiva, entonces, nada parece al azar.

\section{Conclusiones}

Las Maldivas han tenido históricamente y en la actualidad una gran importancia estratégica, a pesar de ser la nación más pequeña de Asia Meridional en términos de población, superficie y PBI. Ello radica en su ubicación en medio de las rutas marítimas internacionales del Océano Índico, y en su cercanía a las costas de la India.

Este artículo se proponía abordar los cambios que se produjeron en Maldivas durante la presidencia de Abdullah Yameen. En cuanto a la política interna, se produjo un deterioro pronunciado de la democracia, con opositores en la cárcel, leyes que dan margen a la arbitrariedad de su aplicación por parte de las fuerzas estatales, etc. (Blomqvist, 2018; Human Rights Watch, 2018; Radhakrishnan, 2016; Ramachandran, 2018b). En cuanto a las relaciones exteriores, el presidente ha estrechado lazos fuertes, inéditos hasta entonces, con la República Popular China, incluyendo una reforma constitucional y un tratado de libre comercio que favorecen el desembarco de la gran nación de Asia del Este en el archipiélago (Blomqvist, 2018; Human Rights Watch, 2018; Radhakrishnan, 2016; Ramachandran, 2018b).

La crisis de 2018 ha puesto de relieve el punto de contacto entre estas dos tendencias. Ante los primeros rumores de la posibilidad de una intervención militar por parte de India, producto de la declaración del estado de emergencia y los disturbios en la capital, China rápidamente salió como garante de la estabilidad del gobierno de Yameen, aduciendo el respeto por la soberanía y la no injerencia en asuntos internos (Rajagopalan, 2018). 
Salvo un cambio fundamental en las circunstancias, parece difícil que el deterioro democrático de Maldivas se detenga. El presidente Yameen continuará acumulando el poder y acrecentando su autoritarismo mientras China le salga de escudo ante toda injerencia externa. A la vez, esto alentará a Yameen a continuar favoreciendo los intereses de China mediante concesiones y privilegios varios.

El desembarco de China en el archipiélago es un asunto de extrema gravedad para los analistas de seguridad en Nueva Delhi, que ya están en alerta por la presencia creciente de los chinos en la región del Océano Índico, en Sri Lanka, Pakistán, entre otros lugares. La reforma constitucional brinda la condición de posibilidad para una futura base china en el archipiélago. Dada la proximidad de las islas al subcontinente indio, este escenario representa una pesadilla estratégica para India (Kumar, 2015; Panda, 2018).

Parece claro que las Maldivas no volverán bajo el ala de India mientras Yameen permanezca en el poder. Las elecciones de 2018, en tanto el oficialismo se ha encargado de impedir la participación efectiva de opositores en la contienda por todos los medios, incluyendo el encarcelamiento y la persecución, no parecen ofrecer una salida.

Durante la crisis de 2018, muchos comentaristas observaron, además, que la regresión democrática en Maldivas es en sí misma un anatema para los intereses estratégicos de largo plazo de India en la Región del Océano Índico, si se tiene en cuenta que India busca posicionarse a sí misma como la guardiana de un Indo-Pacífico "libre y abierto" (Panda, 2018). ¿Qué significa para el estatus y la credibilidad de India ante los otros Estados de su vecindario el hecho de que India no pueda controlar lo que ocurre en una nación pequeña a pocas horas de navegación de sus costas? (Rajagopalan, 2018).

Desde el punto de vista de India, la situación es muy delicada, porque una salida de Yameen solo sería productiva si el sucesor es legítimo, para lo cual tiene que ser un gobierno democrático, pero que al mismo tiempo no sea visto como un títere de India (Dhar, 2018; Ramachandran, 2018b).

En cualquier caso, Maldivas se ha sumado a la lista de puntos estratégicos de la Región del Océano Índico en los cuales India se ve desafiada por la presencia en aumento del poder político y económico de China.

\section{Referencias bibliográficas}

Oliva, C. (2008). Identidad y fuerzas profundas en China. La influencia de la experiencia histórica en la formulación de la política exterior. En A. Busso (Comp.), Fuerzas profundas e identidad. Reflexiones en torno a su impacto sobre la política exterior. Un recorrido de casos (pp. 250-267). Rosario: UNR Editora, e-book. 
Disponible en

https://rephip.unr.edu.ar/bitstream/handle/2133/2578/Fuerzas_Profundas_e_Iden tidad_-_tomo_I.pdf?sequence=1\&isAllowed=y

\section{Otros documentos consultados:}

Blomqvist, O. (2018). Democracy on the Brink in Maldives. The Diplomat (9 de febrero). Recuperado de: https://thediplomat.com/2018/02/democracy-on-the-brink-inmaldives/

Dhar, D. (2018). The Maldives Crisis Presents a Window of Opportunity for India. The Diplomat (25 de febrero). Recuperado de https://thediplomat.com/2018/02/themaldives-crisis-presents-a-window-of-opportunity-for-india/

Green, M. J. (2018). Foreword: China's Maritime Silk Road. En N, Szechenyi (Ed.). China's Maritime Silk Road. Strategic and Economic Implications for the Indo-Pacific Region. CSIS (Marzo). Washington: CSIS.

Human Rights Watch (2018). "An All-Out Assault on Democracy" Crushing Dissent in the Maldives. Disponible en https://www.hrw.org/sites/default/files/report_pdf/maldives0818_web2.pdf

Kabir, A. (2018). The Maldives Crisis: Will India Intervene? The Diplomat (27 de febrero). Recuperado de https://thediplomat.com/2018/02/the-maldives-crisis-will-indiaintervene/

Kaplan, R. D. (2010). Monsoon. The Indian Ocean and the Future of American Power. Nueva York: Random House.

Kumar, S. (2015). "This Will Make the Country a Chinese Colony". The Diplomat (25 de julio). Recuperado de https://thediplomat.com/2015/07/this-will-make-the-countrya-chinese-colony/

Maldives Independent (2018). Indian MP provokes outrage with call to invade Maldives (25 de Agosto). Recuperado de https://maldivesindependent.com/politics/indian-mpprovokes-outrage-with-call-to-invade-maldives-140506

Mohamed, A. (2016). Why Maldives left the Commonwealth. Indian Express (29 de octubre). Recuperado de https://indianexpress.com/article/opinion/columns/why-maldivesleft/

Olazábal, V. (2016). Maldivas dice adiós a la Commonwealth. El Mundo (14 de octubre). Recuperado de 
http://www.elmundo.es/internacional/2016/10/14/5800b7cc268e3ebc518b4586.ht $\mathrm{ml}$

Panda, A. (2018). A Political Crisis Deepens in the Maldives: The Geopolitical Stakes for India and its Options. The Diplomat (6 de febrero). Recuperado de https://thediplomat.com/2018/02/a-political-crisis-deepens-in-the-maldives-thegeopolitical-stakes-for-india-and-its-options/

Pant, H. V. (2018). Trouble in Paradise: How India Can Responded to the Crisis in the Maldives. The Diplomat (7 de febrero). Recuperado de https://thediplomat.com/2018/02/trouble-in-paradise-how-india-can-respond-tothe-crisis-in-the-maldives/

Pararajasingham, A. (2017). India's Regional Power Credentials Under Threat by China. The Diplomat (24 de noviembre). Recuperado de https://thediplomat.com/2017/11/indias-regional-power-credentials-under-threatby-china/

Radhakrishnan, T. (2016). Maldives' Democracy is Dying. The Diplomat (23 de septiembre). Recuperado de https://thediplomat.com/2016/09/maldives-democracy-is-dying/

Rajagopalan, R. P. (2018). India's Maldives Headache. The Diplomat (23 de febrero). Recuperado de https://thediplomat.com/2018/02/indias-maldives-headache/

Ramachandran, S. (2018a). The China-Maldives Connection. The Diplomat (25 de enero). Recuperado de https://thediplomat.com/2018/01/the-china-maldives-connection/ Ramachandran, S. (2018b). India and the Maldives Emergency. The Diplomat (8 de febrero). Recuperado de https://thediplomat.com/2018/02/india-and-themaldives-emergency/

Reddy, M. (2009). Maldives cabinet to meet underwater to highlight perils of climate change. The Hindu (17 de octubre). Recuperado de https://www.thehindu.com/news/international/Maldives-cabinet-to-meetunderwater-to-highlight-perils-of-climate-change/article16887202.ece

Safi, M. (2016). Maldives quits Commonwealth over alleged rights abuses. The Guardian (13 de octubre). Recuperado de https://www.theguardian.com/world/2016/oct/13/maldives-quits-commonwealthover-alleged-rights-abuses

Singh, S. (s.f.). Security Outlook of Indian Ocean and India's Geostrategic interest in the IOR. India Foundation. Recuperado de http://www.indiafoundation.in/security- 
outlook-of-indian-ocean-and-indias-geostrategic-interest-in-the-ior-by-siddharthsingh/

Tiezzi, S. (2018). China to India: Respect Maldives' Sovereignty. The Diplomat (8 de febrero). Recuperado de https://thediplomat.com/2018/02/china-to-india-respectmaldives-sovereignty/ 